\title{
Histopathological Evaluation of Dose Dependent Sulfadiazine-Associated Nephrotoxicity and Alteration on Oxidative Stress in Chicken Embryos
}

\author{
Reza Sayrafi, ${ }^{1}$ Hannaneh Golshahi, ${ }^{2,}{ }^{*}$ Atefeh Araghi, ${ }^{1}$ and Saeed Seifi ${ }^{1}$ \\ ${ }^{1}$ Department of Food Hygiene, Faculty of Veterinary Medicine, Amol University of Special Modern Technologies, Amol, IR Iran \\ ${ }^{2}$ Department of Pathology, Faculty of Veterinary Medicine, University of Tehran, Tehran, IR Iran \\ "Corresponding author: Hannaneh Golshahi, Department of Pathology, Faculty of Veterinary Medicine, University of Tehran, Tehran, IR Iran. E-mail: h.golshahi@ut.ac.ir
}

Received 2015 January 21; Accepted 2015 July 5.

\begin{abstract}
Background: Numerous epidemiological and experimental researches indicate that in utero exposure to some environmental chemicals and prescribed drugs during pregnancy can mediate various embryonic abnormalities and complications via reactive oxygen species (ROS) generation, which damages cellular macromolecules.

Objectives: The aim of the present study was to evaluate the sulfonamide-associated nephrotoxicity with possible underlying mechanisms in chicken embryo.

Materials and Methods: In this experimental study, one hundred fertile eggs were obtained and divided into five groups:1) control group (without injection), 2) group injected with $2 \mathrm{mg}$ sulfadiazine, 3) group injected with $10 \mathrm{mg}$ sulfadiazine, 4) group injected with $30 \mathrm{mg}$ sulfadiazine and 5) group injected with $70 \mathrm{mg}$ sulfadiazine. After hatching, the renal tissue from the newly hatched chick was harvested for histopathologic investigation and also measurement of oxidative stress parameters [the ferric reducing capacity assay, the glutathione content (GSH) and the situation of lipid peroxidation (LPO)] by spectrophotometer.

Results: Histologic examination of the renal tissue revealed that sulfadiazine induces hydropic degeneration, tubular necrosis, glomerular and tubular atrophy, formation of hyaline cast, congestion, hemorrhage, interstitial nephritis and fibrosis.

Conclusions: Result showed the dose-dependent administration of sulfadiazine significantly altered the histopathologic structure of renal tissues of chickens. Furthermore, the major histopathologic events in the course of sulfadiazine cytotoxicity are renal tubule epithelial cell necrosis, interstitial nephritis and fibrosis, formation of hyaline cast and congestion and hemorrhage, although sulfadiazine at dose $30 \mathrm{mg}$ and $70 \mathrm{mg}$ caused perturbation in antioxidant defense system by marked increase in LPO, and decrease in GSH.
\end{abstract}

Keywords: Sulfadiazine, Kidney, Chicken, Toxicity, Embryo Developmental

\section{Background}

Sulfonamides are relatively old synthetic antibacterial compounds, which can inhibit both Gram-positive and Gram-negative bacteria, as well as some protozoa, such as coccidials [1]. Sulfonamides can interfere with multiplication of the bacterial cell by completely competitively binding the para aminobenzoic acid (PABA) and prevent the folic acid formation, an important metabolite in DNA synthesis. Therefore, sulfonamides are usually bacteriostatic $[2,3]$. Because of low cost, ease of administration, and a wide range of application caused the extensive use of sulfonamide derivatives and resulting in a rapid rise of bacteria-resistance, cross-resistance among sulfonamides and residues appearing in animal products $[1,4,5]$. The concentrations of residues are varying considerably in various tissues and the highest level exists in body fats and/or in organs that actively metabolize and excrete them espe- cially liver and kidney [5, 6].

In poultry science, sulfonamides have been used for treating of various diseases like cocidiosis, infectious coryza, pullorum disease and flow typhoid [1]. As reported previously, following the treatment of infected hen with sulfonamides, the residues of them are also retained in the chicken and egg layers [7]. Studies revealed that the presence of sulfonamides residues in food is considered harmful to consumers [5]. These have adverse reactions such as renal insufficiency side effects $[8,9]$.

\section{Objectives}

In this present study, we investigated the effects of the administration of different doses of sulfadiazine in chicken embryo as an experimental model in order to detect histopathologic alterations between low and high doses. According to the author's knowledge, there is no 
information currently available about the sulfonamideassociated nephrotoxicity with possible underlying mechanisms in chicken embryo, and the present study is the first attempt in this field.

\section{Materials and Methods}

This study was conformed to the rules of the Protection of vertebrate animals used for experimental and other scientific purposes [10]. In this experimental study, one hundred fertile eggs were obtained from a broiler breeder farm (Ross 308 strain). All eggs weighed with an average of $63 \pm$ $1 \mathrm{~g}$ were divided into five groups: 1 ) control group (without injection), 2) group injected with $2 \mathrm{mg}$ sulfadiazine, 3 ) group injected with $10 \mathrm{mg}$ sulfadiazine, 4) group injected with $30 \mathrm{mg}$ sulfadiazine and 5) group injected with $70 \mathrm{mg}$ sulfadiazine. Then, the eggs were incubated at $37.5^{\circ} \mathrm{C}$ and $65 \%$ relative humidity. On third day of incubation, the eggs were candled, clear eggs and dead embryos were removed for examination. Then shortly after, the eggs were injected in ovo at fourth day of incubation via chorioallantoic rout with $0.2 \mathrm{~mL}$ of mentioned doses. The injection site was sealed with Betadine ${ }^{\circledR}$. To avoid contamination, all injections were carried out in a clean room and all the equipments were sterilized. On the eighteen day of incubation, the fertile eggs were transferred to the hatcher and kept at a temperature of $37^{\circ} \mathrm{C}$ until they hatch.

During the incubation, dead embryos were removed ( $n$ =9). After hatching, the kidney from the newly hatched chick was taken out $(\mathrm{n}=30)$ and fixed in $10 \%$ formalin for histological examination. Some of kidneys $(n=80)$ were also stored at $-70^{\circ} \mathrm{C}$ until used for assessing oxidative stress examinations. The isolated whole kidney tissue was homogenized with 10 times (w/v) sodium phosphate buffer. The homogenate was centrifuged at 3,000 rpm for $15 \mathrm{~min}$ utes, and the supernatant was used for estimation of biochemical indices.

Histopathologic examination: Following histological fixation, the renal tissues were dehydrated by transferring through a series of alcohols with increasing concentrations, placed into xylol and embedded in paraffin. A microtome was used to make 8 cuts that were 6-7 $\mu \mathrm{m}$ and they were stained with hematoxylin-eosin ( $\mathrm{H} \& \mathrm{E})$. The sections were observed under light microscope. Histopathologic changes in renal tissues were graded as follows: (-) showing no changes, $(+),(++)$ and $(+++)$ indicating mild, moderate and severe changes, respectively.
3.1. Measurement of Oxidative Stress Parameters in Organ System

\subsubsection{The Ferric Reducing Capacity Assay}

The antioxidant capacity of samples was determined by measuring the ability of samples to reduce $\mathrm{Fe}^{3+}$ to $\mathrm{Fe}^{2+}$. The complex between $\mathrm{Fe}^{2+}$ and 2, 4, 6-tris-(2-pyridyl)-1, 3, 5triazine (TPTZ) gives a blue color with absorbance at 593 $\mathrm{nm}$ [11].

\subsubsection{Measurement of Reduced Glutathione (GSH)}

The glutathione content was applied according to the previous method [12]. Briefly, the cells were rinsed three times with phosphate-buffered saline (PBS). The cell solution mixed with $20 \%$ trichloroacetic acid. Samples were centrifuged at $3000 \mathrm{rpm}$ for 15 minutes. The supernatant was mixed with 4 volumes of Tris. Then, 1 mM 5, 5'-dithiobis (2-nitrobenzoic acid) (DTNB) was added to the sample and incubated for 30 minutes. The absorbance was read at 412 $\mathrm{nm}$.

\subsubsection{Measurement of Lipid Peroxidation}

The formation of thiobarbituric acid in organ samples was assessed for the measurement of lipid peroxidation according to an original method [13]. Briefly, the supernatant of the tissue homogenate was mixed with $20 \%$ trichloroacetic acid and the mixture was centrifuged. Then, thiobarbituric acid was added to the supernatant and heated. The absorbance of the supernatant was measured at $532 \mathrm{~nm}$. The values were expressed in nmoles (nM) malodialdehyde (MDA), using a molar extinction coefficient of $1.56 \times 10^{5} \mathrm{M}^{-1} \mathrm{~cm}^{-1}$.

Oxidative stress data were analyzed using one way ANOVA using SPSS-16 software followed by Tukey-Kramer post-hoc test for multiple comparisons. Kolmogorov Smirnov tests showed that these data were normally distributed. The evaluation was made by the comparison of groups. The results were presented as means \pm SEM and $\mathrm{P}$ $<0.05$ was considered significant.

\section{Results}

Histological examination of the kidney tissues in the control group revealed no significant deviation from the normal histological structures. In group 2, hydropic degeneration was observed in epithelial cells of the tubules. No evidence of tubular epithelial necrosis was seen. Within the lumen of collecting tubules, fan-shaped, empty spaces indicated sulfadiazine crystals. In addition, hyaline casts were seen in the lumen of some tubules. In general, pathological changes, formed by administration of $2 \mathrm{mg}$ of sulfadiazine, were mild (Table 1). In group 3, the pathological changes compared with those in the group 2 were 
very common. However, hydropic degeneration (Figure 1) was more severe than group 2 and also, some apoptotic epithelial cells, mild multifocal non-supportive interstitial nephritis and congestion has been found in this group (Table1). Fan-shaped, empty spaces representing the spaces once occupied by sulfadiazine crystals had been often present (Figure 2). Hyaline casts were seen in the lumen of some tubules. In group 4, hydropic degeneration was more severe compared to previous groups. Fragmentation and shedding of tubular epithelium that indicated cellular necrosis were significantly identified in this group. Some Bowman's spaces and tubules showed dilation. The severity of interstitial nephritis was more than group 3. Congestion was more severe compared to previous groups (Table 1), and also, some foci of hemorrhage were observed in the renal interstitium. In group 5, epithelial necrosis was more severe compared to group 4. Fan-shaped, empty spaces accumulated in the lumen of collecting tubules. Multifocal interstitial nephritis was reported (Figure 3). Mild fibrosis areas and hyaline cast were observed in the lumen of many tubules (Figure 4). Congestion and hemorrhage were observed in the cortical and medullary interstitial areas. Some glomerulus showed atrophic change and also dilation of Bowman's spaces (Table 1).

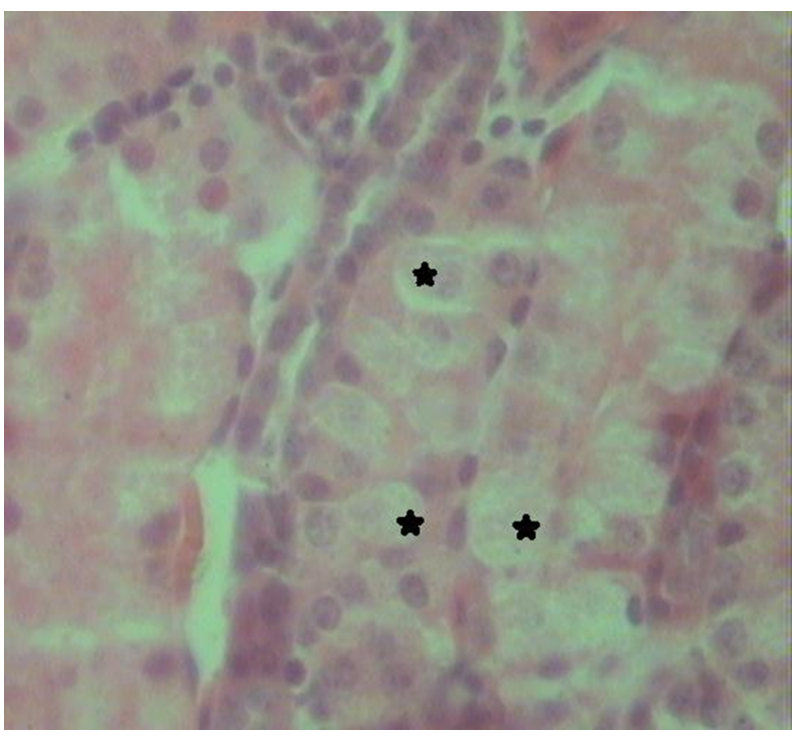

Figure 1. Hydropic Degenerations Were Showed in the Cytoplasms of Epithelial Cells of Tubules (Star) (H \& E 400x)

Oxidative stress results have illustrated in Table 2. There was no significant difference between the levels of antioxidant in kidney of group 1and 2 compared to the control group, but in high dose group (4 and 5) antioxidant level was lower than control and other groups, $(\mathrm{P}=0.03)$

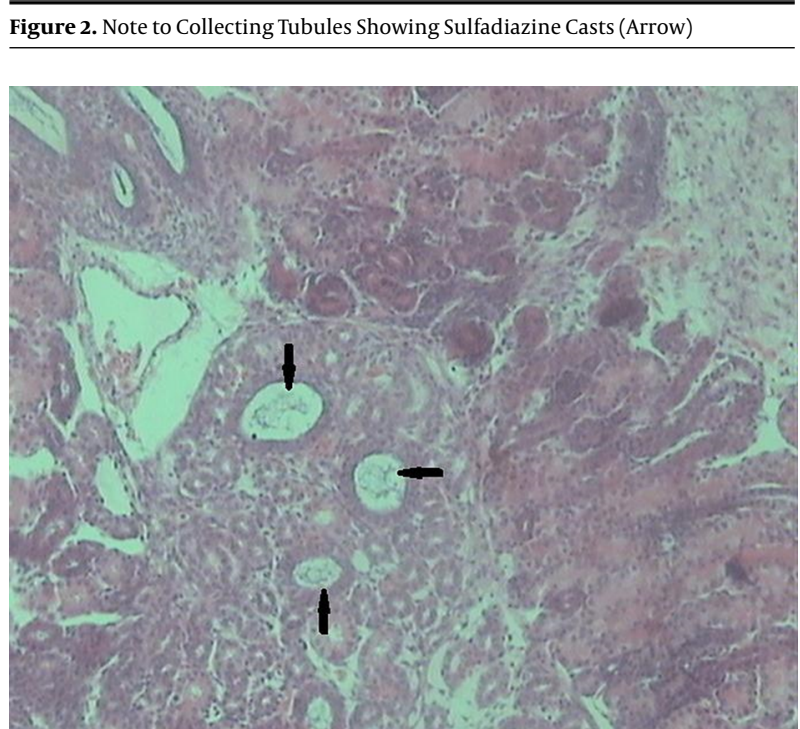

Spaces are left in cast after sulfadiazine is dissolved (H \& E 40x)

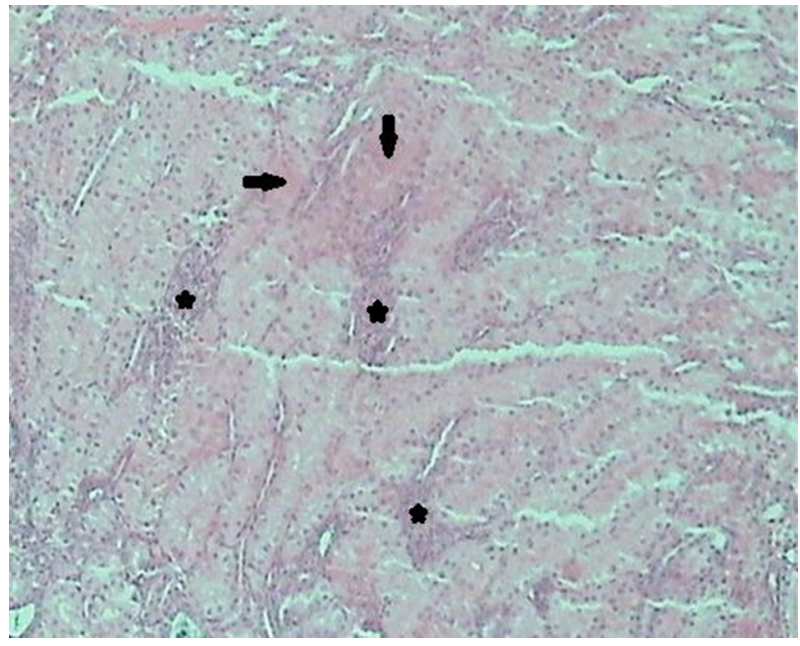

Figure 3. Note to Tubular Necrosis (Arrow) and Mononuclear Interstitial Nephritis (Stars) in the High Dose Administered Group (Group 5) (H \& E, 100x)

for group 4 vs. control group, $(P=0.01)$ for group 5 vs. control group and $(\mathrm{P}=0.02)$ for group 5 vs. group 2 .

The levels of malodialdehyde (MDA) in kidney were higher in group 4 and 5 vs. control group $(\mathrm{P}=0.001)$, group 2 vs. group $4(\mathrm{P}=0.0001)$, group 3 vs. group $4(\mathrm{P}=0.05)$, group 2 vs. group $5(\mathrm{P}=0.001)$, group3 vs. group $5(\mathrm{P}=$ 0.001).

GSH of the kidney in experimental groups was significantly lower than the control in group 3, 4 and $5(\mathrm{P}=0.03)$ for group 3 vs. $\mathrm{C}$ group and $(\mathrm{P}=0.01)$ for group 4 and group 5 vs. C group. 
Table 1. Histopathologic Changes Induced by Different Doses of Sulfadiazine in Renal Tissue ${ }^{\text {a }}$

\begin{tabular}{|c|c|c|c|c|c|}
\hline \multirow[b]{2}{*}{ Parameters } & \multicolumn{5}{|c|}{ Groups } \\
\hline & Group 1 & Group 2 & Group 3 & Group 4 & Group 5 \\
\hline Tubular epithelial cell degeneration & - & + & ++ & +++ & +++ \\
\hline Tubular epithelial cell necrosis & - & - & - & + & +++ \\
\hline Atrophic glomerulus and tubules & - & - & - & - & + \\
\hline Fan-shaped, empty spaces & - & + & ++ & +++ & +++ \\
\hline Hyaline cast in the tubule lumen & - & + & + & ++ & +++ \\
\hline Congestion in vessels of the interstitial tissue & - & - & + & ++ & +++ \\
\hline Interstitial mononuclear cell infiltration & - & - & + & ++ & +++ \\
\hline Increased fibrous tissue & - & - & - & - & + \\
\hline
\end{tabular}

${ }^{a}$ Histopathologic changes in renal tissues were graded as follows: (-) showing no changes, $(+),(++)$ and (+++) indicating mild, moderate and severe changes, respectively.

Table 2. Oxidative and Antioxidant Parameters in Chicken Embryos Were Exposed to Different Doses of Sulfadiazine $\mathrm{a}^{\mathrm{a}, \mathrm{b}, \mathrm{c}, \mathrm{d}, \mathrm{e}, \mathrm{f}}$

\begin{tabular}{|c|c|c|c|c|c|}
\hline & Group 1 (Control) & Group 2 & Group 3 & Group 4 & Group 5 \\
\hline Total antioxidant activity (FRAP) & $1.89 \pm 0.24$ & $1.62 \pm 0.17$ & $1.37 \pm 0.14$ & $1.13 \pm 0.09^{\mathrm{A}^{*}}$ & $0.9 \pm 0.05^{A^{* s}, B^{*}}$ \\
\hline Malodialdehyde (MDA) & $0.53 \pm 0.16$ & $0.66 \pm 0.19$ & $1.01 \pm 0.27$ & $1.72 \pm 0.53^{\mathrm{A}^{* * *}, \mathrm{C}^{* * *}, \mathrm{D}^{*}}$ & $2.03 \pm 0.43^{\mathrm{A}^{* * *}, \mathrm{~B}^{* * * *}, \mathrm{E}^{* * *}}$ \\
\hline Intracellular GSH & $0.07 \pm 0.03$ & $0.04 \pm 0.03$ & $0.03 \pm 0.02^{\mathrm{A}^{*}}$ & $0.02 \pm 0.01^{\mathrm{A}^{* *}}$ & $0.02 \pm 0.00^{\mathrm{A}^{* *}}$ \\
\hline
\end{tabular}

${ }^{a}$ Statistical significance for difference the between the data of $C$ groups vs. groups $2,3,4$ and $5 ; A^{*}: P=0.03, A^{* *}: P=0.01, A^{* * *}: P=0.001$.

${ }^{b}$ Statistical significance for difference the between the data of group 2 vs. group $5 ; B^{*}: P=0.04, B^{* * *}: P=0.001$.

${ }^{c}$ Statistical significance for difference the between the data of group 2 vs. group $4 ; C^{*}: P=0.045, C^{* * *}: P=0.001$.

${ }^{\mathrm{d}}$ Statistical significance for difference the between the data of group 3 vs. group $4 ; D^{*}: P=0.05, D^{* *}: P=0.04, D^{* * *}: P=0.001$.

${ }^{\mathrm{e}}$ Statistical significance for difference the between the data of group 3 vs. group $5 ; \mathrm{E}^{*}: \mathrm{P}=0.04, \mathrm{E}^{* * *}: \mathrm{P}=0.001$.

${ }^{\mathrm{f}}$ Values are expressed as mean $\pm \mathrm{SD}$.

\section{Discussion}

The current study shows that pathologic lesions in the kidney are responsible for treatment with sulfonamide. Histopohatlogical examination of the renal tissue revealed that sulfadiazine induces hydropic degeneration, tubular necrosis, glomerular and tubular atrophy, formation of hyaline cast, congestion, hemorrhage, interstitial nephritis and fibrosis. In the present study, dose-dependent sulfadiazine-associated nephrotoxicity has been identified. In the group 2 taken $2 \mathrm{mg}$ sulfadiazine; changes were pretty mild and reversible. In group 3 with administration of $10 \mathrm{mg}$ sulfadiazine, the changes were more severe though reversible yet. Group 4 and 5 received $30 \mathrm{mg}$ and $70 \mathrm{mg}$ sulfadiazine respectively. High dose administration caused both reversible and irreversible pathological changes with more severity compared to control and other treated groups. However, in group 5, irreversible injuries were more severe and increased fibrous tissue was noted.

Majeed et al. [9] studied the toxicological effect of sulfonamide in domestic pigeons by oral intubation of two dosage levels as intermediate $40 \mathrm{mg} / \mathrm{kg}$ and high $80 \mathrm{mg} / \mathrm{kg}$.
Histopathological results demonstrated nephrotoxic effects characterized by degenerate and/or dilated cortical tubules in intermediate while, high dose groups appeared glomerular atrophy some with dilated Bowman's spaces, the severity of the changes were more intense in the high dose group, though the changes did not lead to necrosis.

In another study, Islam et al. [8] investigated the counteracting effect of Spirulina against potentiated sulfonamides side effects in rats. Resulting significant histopathological changes in the kidney of treated rats with sulfonamide (96 mg/rat/day) throughout the experimental period of 60 days, represent by of slight degenerative in the renal parenchyma. These results were compatible with the present study except we detected irreversible injuries like tubular necrosis in high dose administration (30 and $70 \mathrm{mg}$ ), and also, in our study, embryos received a single dose of sulfadiazine. Odigie [14] investigated the morphological alteration of visceral organs (kidney and liver) of albino Wister rats pre-exposed to prophylactic consumption of sulfonamide based drugs. Histological findings indicated that oral treatment by 3 and $4 \mathrm{mg}$ drug for 21 days caused infiltration of inflammatory cells, congestion 


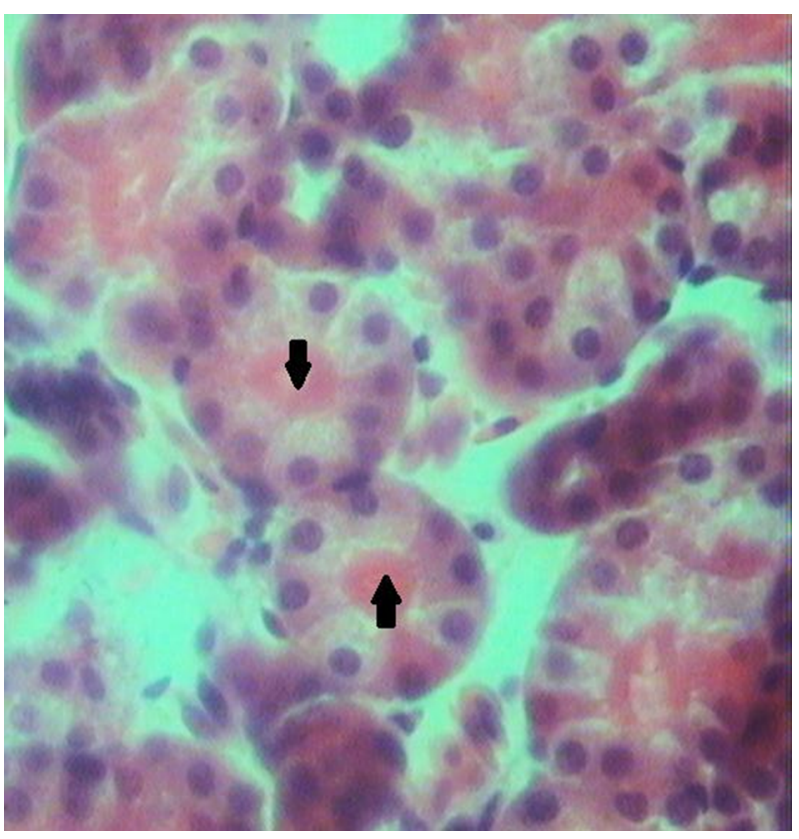

Figure 4. Note to Hyaline Casts in Proximal Convoluted Tubules (Arrow) (H \& E, 400x)

of glomerulus, hematomas, thickening of the interstitial cells and vacuolation with congestive and tubular necrosis as compared to the control group. However, in present cases, chicken embryos received $2 \mathrm{mg}$ and $10 \mathrm{mg}$ by in ovo injection, showed mild pathological changes.

Lebkowska-Wieruszewska and Kowalski [15] evaluated the residue depletion in healthy turkeys treated with sulfachloropyrazine. Results showed that sulfachloropyrazine has a long half-life and relatively high bioavailability. The drug was found in measurable edible tissues of turkeys eighteen days after the cessation of treatment.

Haritova et al. [3] studied pharmacokinetics of sulfachlorpyrazine-sodium in healthy chickens and chickens experimentally infected with Eimeria tenella. Results showed that pathological changes in E. tenella infected chickens contribute to slower absorption and elimination rate of sulfachlorpyrazine which resulted in higher accumulation of the drug in the body.

Malik et al. [5] studied effect of sulfonamides residues on egg quality traits resulting sulfonamide drug affected the external and internal quality of egg by decreasing egg shell weight, shell thickness, yolk height, yolk width and also yolk index. In this regard, it is suitable to escape eggs selling and consumption during treatment and withdrawal periods. The presence of sulfonamide residues necessitates the application of biosecurity measures at poultry farm level.
In oxidative stress, MDA content, has been increased by the higher concentration of sulfadiazine, on the other hand the lower concentrations were on the contrary. As shown in table 2 embryos that received sulfadiazine at dose $30 \mathrm{mg}$ and $70 \mathrm{mg}$ showed significant increase in MDA in the kidney tissue as compared to the control animals and group $2 \mathrm{mg}$ and $10 \mathrm{mg}$ sulfadiazine. These results were compatible by histopathological results.

GSH has been accepted as a ubiquitous sulfhydrylcontaining molecule in cells that it is responsible for maintaining cellular oxidation-reduction homeostasis. Alterations in GSH homeostasis can be considered as an indication of functional-damage to the cells [16]. As shown in Table 2 sulfadiazine at dose $10 \mathrm{mg}, 30 \mathrm{mg}$ and $70 \mathrm{mg}$ decreased GSH levels in the cells in a concentration dependent manner. Therefore, it can be assumed that the reduction in GSH concentration might cause the effectiveness of GST and GPx activity to be restricted, as evident by the intensification of lipid peroxidation [17]. We suspected that the observed increased concentration of lipid peroxides, along with decreased GSH was capable of inducing some injuries such as apoptosis visible at the cellular level. Concomitant cellular oxidative stress was revealed by reduced GSH levels, and amplified lipid peroxidation [16].

Oxidative stress (OS) plays a significant role in the pathogenesis of renal disease and its progression [18]. Kidney damage in OS-related Acute Kidney Injury (AKI) was associated with increased reactive oxygen species (ROS) production, leading to oxidation of several macromolecules (e.g. protein, DNA and lipid). Production of lipid peroxidation (LPO) in OS-related AKI results in large production of secondary products such as malondialdehye (MDA) and 4-hydroxynonenal $[19,20]$.

In conclusion, we have demonstrated that, dosedependent administration of sulfadiazine significantly altered the histopathologic structure of renal tissues of chickens. Furthermore, the major histopathologic events in the course of sulfadiazine cytotoxicity are renal tubule epithelial cell necrosis, interstitial nephritis and fibrosis, formation of hyaline cast and congestion and hemorrhage. And also, sulfadiazine at dose $30 \mathrm{mg}$ and $70 \mathrm{mg}$ caused perturbation in antioxidant defense system by marked increase in LPO, and decrease in GSH.

\section{Acknowledgments}

We are thankful from Amol University of Special Modern Technologies that confirmed this research (project No: 94-20) by Reza Sayrafi. 


\section{Footnotes}

Authors' Contribution: Reza Sayrafi, Atefeh Araghi and Saeed seifi designed the study and carried out the experiments. Hannaneh Golshahi and Reza Sayrafi participated in the histopathological evaluation, acquired photomicrographs and gave the final histopathological diagnosis. Atefeh Araghi performed biochemical analysis and contributed to the interpretation of the data. Hannaneh Golshahi and Atefeh Araghi performed the literature review and wrote the manuscript. All authors read and approved the final manuscript.

Funding/Support: Faculty of Veterinary Medicine, Amol University of Special Modern Technologies.

\section{References}

1. Prescott JF, Dowling PM. Antimicrobial therapy in veterinary medicine. John Wiley \& Sons; 2013.

2. Greif G, Harder A, Haberkorn A. Chemotherapeutic approaches to protozoa: Coccidiae-current level of knowledge and outlook. Parasitol Res. 2001;87(11):973-5. [PubMed:11728025].

3. Haritova AM, Lashev LD, Koinarski VC. Sulfachloropyrazine disposition in Eimeria tenella infected chickens. Veterinarski Arhiv. 2013;83(2):211-22.

4. Smith JL, Drum DJ, Dai Y, Kim JM, Sanchez S, Maurer JJ, et al. Impact of antimicrobial usage on antimicrobial resistance in commensal Escherichia coli strains colonizing broiler chickens. Appl Environ Microbiol. 2007;73(5):1404-14. doi:10.1128/AEM.01193-06. [PubMed: 17194843].

5. Malik HEE, Omer JE, Elamin KM. Effect of Sulfanomides Residues on Egg Quality Traits. Int J Poultry Sci. 2013;12(5):312.

6. Booth NH. Development of a regulatory and research program in veterinary medical toxicology. J Vet Toxicol. 1973;15:100-3.

7. Mehtabuddin A, Ahmad T, Nadeem S, Tanveer Z, Arshad J. Sulfonamide residues determination in commercial poultry meat and eggs.JAnim Plant Sci. 2012;22(2):473-8.

8. Islam M, Akter S, Bala S, Hossain MZ, Akter MS. Investigation on the counteracting effect of spirulina against potentated sulfonamide's (cotrim ds®) side effects in rat. Bangladesh JVet Med. 2013;10(1-2):81-6.
9. Majeed SK, Al-Sereah BA, Essa IM. The sulfonamide exposure and the histopathological alterations of domestic pigeon. Bas J Vet Res. 2014;1(1):82-93.

10. Multilateral Consultation . Appendix A of the European convention for the protection of vertebrate animals used for experimental and other scientific purposes (ETS No.123), guidelines for accommodation and care of animals (Article 5 of the Convention) 1986. Available from: https://www.aaalac.org/about/AppA-ETS123.pdf.

11. Benzie IF, Strain JJ. The ferric reducing ability of plasma (FRAP) as a measure of "antioxidant power": the FRAP assay. Anal Biochem. 1996;239(1):70-6. doi: 10.1006/abio.1996.0292. [PubMed: 8660627].

12. Gibson XA, Shartava A, McIntyre J, Monteiro CA, Zhang Y, Shah A, et al. The efficacy of reducing agents or antioxidants in blocking the formation of dense cells and irreversibly sickled cells in vitro. Blood. 1998;91(11):4373-8. [PubMed: 9596687].

13. Sicinska P, Bukowska B, Michalowicz J, Duda W. Damage of cell membrane and antioxidative system in human erythrocytes incubated with microcystin-LR in vitro. Toxicon. 2006;47(4):387-97. doi 10.1016/j.toxicon.2005.12.006. [PubMed:16457864].

14. Odigie BE. Histological effects of pre-exposure prophylactic consumption of sulfa drugs on Liver and Kidney of albino Wister rats (Rattus novergicus).J Pharm Biol Sci. 2013;5(6):14-9.

15. Lebkowska-Wieruszewska BI, Kowalski CJ. Sulfachlorpyrazine residues depletion in turkey edible tissues. $J$ Vet Pharmacol Ther 2010;33(4):389-95. doi: 10.1111/j.1365-2885.2009.01147.x. [PubMed: 20646202].

16. Reddy AR, Reddy YN, Krishna DR, Himabindu V. Multi wall carbon nanotubes induce oxidative stress and cytotoxicity in human embryonic kidney (HEK293) cells. Toxicology. 2010;272(1-3):11-6. doi: 10.1016/j.tox.2010.03.017. [PubMed: 20371264].

17. Yousef MI, Omar SA, El-Guendi MI, Abdelmegid LA. Potential protective effects of quercetin and curcumin on paracetamol-induced histological changes, oxidative stress, impaired liver and kidney functions and haematotoxicity in rat. Food Chem Toxicol. 2010;48(11):324661. doi: 10.1016/j.fct.2010.08.034. [PubMed: 20804811].

18. Haugen E, Nath KA. The involvement of oxidative stress in the progression of renal injury. Blood Purif. 1999;17(2-3):58-65. [PubMed: 10449863].

19. Cristol JP, Thiemermann C, Guerin MC, Torreilles J, de Paulet AC. LArginine infusion after ischaemia-reperfusion of rat kidney enhances lipid peroxidation. J Lipid Mediat Cell Signal. 1996;13(1):9-17. [PubMed 8821807].

20. Palipoch S. A review of oxidative stress in acute kidney injury: protective role of medicinal plants-derived antioxidants. Afr J Tradit Complement Altern Med. 2013;10(4):88-93. [PubMed: 24146507]. 